\title{
SMASH: A Distributed Sensing and Processing Garment for the Classification of Upper Body Postures
}

\author{
Holger Harms \\ Wearable Computing Lab. \\ ETH Zurich \\ harms@ife.ee.ethz.ch
}

\author{
Oliver Amft \\ Wearable Computing Lab. \\ ETH Zurich \\ amft@ife.ee.ethz.ch \\ Gerhard Tröster \\ Wearable Computing Lab. \\ ETH Zurich \\ troester@ife.ee.ethz.ch
}

\author{
Daniel Roggen \\ Wearable Computing Lab. \\ ETH Zurich \\ roggen@ife.ee.ethz.ch
}

\begin{abstract}
This paper introduces a smart textile for posture classification. A distributed sensing and processing architecture is implemented into a loose fitting long sleeve shirt. Standardized interfaces to remote periphery support the variable placement of different sensor modalities at any location of the textile.

The shirt is equipped with acceleration sensors in order to determine the postural resolution and the systems feasibility for applications in movement rehabilitation. For the garment characterization an arm posture measurement method is proposed and applied in a study with 5 users.

The classification performance is analyzed on data from overall 8 users, conducting 12 posture types, relevant for shoulder and elbow joint rehabilitation. We present results for different user-modes, with classification rates of $89 \%$ for a user-independent evaluation. Moreover, the relation of body dimensions on the posture classification performance are analyzed.
\end{abstract}

\section{Keywords}

Smart Shirt, SMASH, Smart Textiles, Posture Classification

\section{INTRODUCTION}

Posture classification is an essential basis for activity recognition in various health-related applications. These include virtual assistants for movement rehabilitation to regain movement flexibility, or coaching support to maintain favorable upper body postures during daily activities. The common vision for virtual movement assistants is to empower the user with preventive coaching to reduce risk of hospitalization and chronic diseases and track rehabilitation progress.

On-body sensing of postures has grown to a first-choice solution for these applications, since the sensors are cheap and

Permission to make digital or hard copies of all or part of this work for personal or classroom use is granted without fee provided that copies are not made or distributed for profit or commercial advantage and that copies bear this notice and the full citation on the first page. To copy otherwise, to republish, to post on servers or to redistribute to lists, requires prior specific permission and/or a fee.

BodyNets 2008, March 13-15 Tempe, Arizona, USA

Copyright $\odot 2008$ ICST 978-963-9799-17-2

DOI 10.4108/ICST.BODYNETS2008.2955 lightweight and do not require complex room setups. However, sensors and electronics need to be unobtrusive for a seamless integration to the body. The large amount of sensor data must be reduced in order to allow off-body transmissions, e.g. to a therapy computer or for user feedback. This typically requires an online recognition of relevant postures. Sensor and on-body processing must satisfy the stringent power and robustness constraints for daylong use of wearable systems. While different wearable sensing approaches have been proposed, few of the works actually present a full system solution for on-body sensing and recognition that fulfill these requirements.

In this paper, we present the design and implementation of a novel posture and movement sensing platform, called SMASH (SMArt SHirt), that is integrated into a not specifically tightened textile. SMASH is designed to operate as a comfortable monitoring garment for everyday use in movement rehabilitation or sports coaching. Our approach follows the concept, that sensing and processing tasks can be efficiently implemented using a distributed system architecture.

To this end, the paper presents the following contributions:

1. We introduce SMASH and describe the garment's sensing and hierarchical data processing architecture. The latter resembles three processing layers: sensor data acquisition, feature processing and classification. Sensing terminals are connected to a textile integrated core system, consisting of interface gateways and a central system master. The master performs classification tasks on the preprocessed data from the gateways.

2. We present a characterization procedure to analyze the SMASH system. With this approach, the system's resolution is evaluated for arm postures with five users. The posture resolution is derived from the absolute measurement error and verified by classifying 37 arm angle postures.

3. We show that the system can be applied in movement rehabilitation of shoulder and elbow joints. Here we present a study with eight healthy individuals wearing the garment and performing 12 relevant postures 
(see Figure 8). We achieved good classification rates for different training modes, using simple acceleration sensors instead of full inertial systems. In addition, we discuss the garment's feasibility with regard to errors incurred from the non-tightened fitting.

In the following section we highlight different related approaches and show the advantages of the SMASH system. Section 2 outlines the distributed system architecture along with its garment implementation. Sections 3 and 4 present our characterization and movement rehabilitation investigations respectively. Finally, Section 5 summarizes the results of this work.

\subsection{Related Work}

Textile-based posture sensing solutions have been investigated for different sensors and target applications. Major research areas include the reconstruction of hand gestures with glove-attached accelerometers [1], conductive elastomers [2] or cameras $[3,4]$. One pioneering project for upper body monitoring was the Georgia Tech Wearable Motherboard [5]. The system uses optical fibers to detect bullet wounds and other sensor modalities to monitor a soldier's vital conditions in combat conditions.

Several investigators attached sensors onto tight fitting clothes in order to determine postures of the upper body. Dunne et al. [6] developed a garment-integrated plastic optical fiber for monitoring seated spinal postures in one dimension. Tognetti et al. [2] investigated a conductive elastomer, that shows piezoresistive properties when it is deformed. The material was applied in a special layout pattern to the shoulder, elbow and wrist region of a upper limb kinesthetic garment shirt. Thereon, the shirt was used by Giorgino et al. [7] for posture classification. Mattmann et al. [8] analyzed a novel elongation-sensitive yarn and classified upper body postures with a tight fitting suit called Backmanager.

The latter two approaches used textile attached or integrated strain sensors, relying upon the hypothesis, that different postures result in distinguishable elongation patterns. An integration of strain sensors fixes application to specific body regions. For the processing of sensor data, typically off-body computers are utilized. However, monitoring applications in rehabilitation and sports require free movement and on-body processing capabilities.

Junker et al. [9] implemented PadNET, a wearable data acquisition platform for the upper body. PadNET consists of a multistage sensor network that was integrated into a jacket. Sensors were connected by woven wires and placed into pockets. A crucial drawback is the bandwidth limitation of the system bus. The system proposed here, shrinks the area for garment attached sensing platforms by factor 12.5 compared to PadNET.

Gyroscopes and accelerometer-magnetometer pairs are wellestablished sensing solutions for posture tracking. The complementary nature of the sensors was exploited in academia and industry. Several approaches were made to determine the orientation of stand-alone sensor units using Kalman filtering $[11,12,13]$. All these academic investigations are not textile and rely on a defined number and location of sensors.
Moven [10] is a commercially tight fitting motion capturing system for short, but precise recordings. It consists of up to 16 body worn inertial motion sensing units and two masters, connected by a wireless link to a host PC. While these sensing solutions are very precise, the size and power consumption renders the devices infeasible for daylong recordings. Consequently, our approach relies on small and low-power accelerometers only.

\section{SYSTEM ARCHITECTURE}

This chapter provides a system overview and discusses design issues. Afterwards, all units of SMASH are described in detail.

\subsection{Overview and Terms}

SMASH is made of a hierarchical processing network. The central system master, the Konnex, is connected to four Gateways by a wired system bus. Together they form the core system, which is fully integrated to the textile. Each of the four Gateways provides standardized interfaces to outer peripheral platforms, called Terminals. One kind of Terminal is the acceleration Terminal.

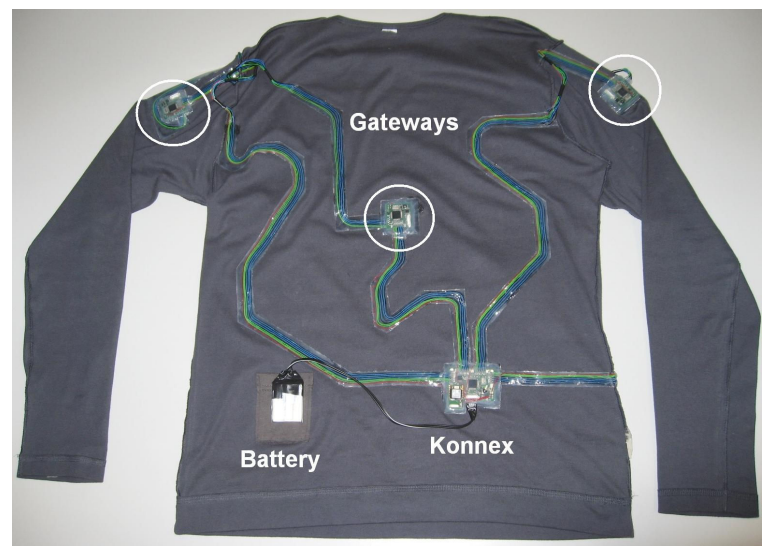

Figure 1: The SMASH (inside out) showing the Konnex and three Gateways (each is marked with a circle, the fourth Gateway is hidden).

\subsection{System Design}

SMASH was designed to acquire, evaluate and process signal data, perform an online classification and to report results to the user. In this flow, information are represented on different levels of abstraction, like physical observations, electrical signals, meaningful features and interpreted classification outputs. One of our main design goal for SMASH was the distribution of processing tasks onto different computation units in a hierarchical way. Hence, we decided to separate and process data in three layers according to their level of abstraction: signals, features, classification. An overview is depicted in Figure 2.

Terminals convert physical observations into an electrical signal representation. Signals are filtered and translated into a standardized format for further processing. However, Terminals act on signal level and are the first processing layer. Afterwards, the Terminal sends collected data to one of the Gateways over a wired connection. Gateways acquire data 


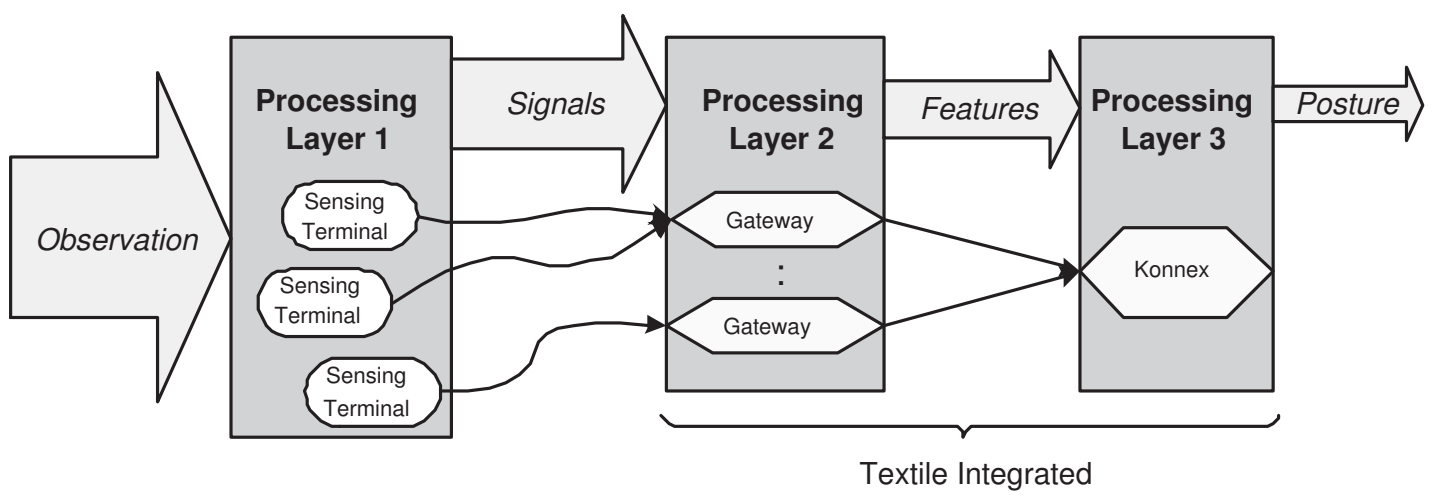

Figure 2: Dependence between the information flow and the system architecture.

from several attached Terminals and fuse them in order to extract meaningful features. Gateways are the second processing layer on the feature level. Gateways send features to the Konnex, which is the last processing layer. It takes the features as an input for an online classification and reports the results to the user. Especially in case of the terminals it was the aim to achieve a minimum PCB size. Hence, all electronic components were selected after their chip area.

Posture classes are discriminated on the Konnex by a Nearest Centroid Classifier. The unit is able to perform a samplewise realtime classification with seven active acceleration Terminals and a sample frequency of $16 \mathrm{~Hz}$. SMASH can alternatively be configured to operate as a pure data acquisition hardware system. Gathered sensor data can be either stored into a non-volatile memory or sent to an outer host via an integrated IEEE 802.15.4 compatible Bluetooth module. Locally developed data recording software receives, visualizes and stores received data.

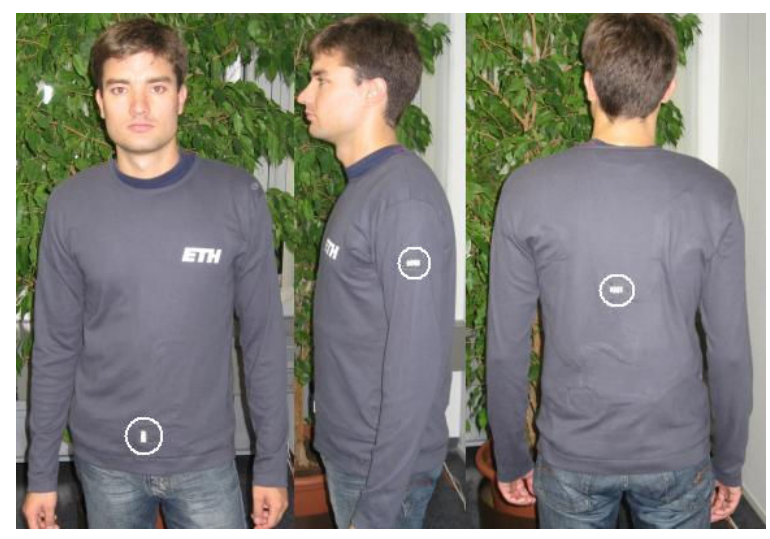

Figure 3: The worn SMASH. White bars at the upper arm, back and lower waist are the Gateway's connectors to Terminals (marked with a circle).

\subsection{Terminals}

Terminals are miniature and lightweight sensors or actuators, connected to the core system via a 2 -wire $I^{2} C$ bus (see Figure 4). SMASH is able to detect plugged Terminals and identify their services during runtime. For this, the available $I^{2} C$ address space was segmented, each segment is reserved for one type of Terminal. Gateways are polling the available address space by sending a short ping-message to every address. Newly connected Terminals respond to that ping-message and, hence, are identified. Each implemented kind of Terminal is equipped with an ATmega48 8-bit microprocessor for basic pre-processings. It offers all required functionality and interfaces with a size of $5 \times 5 \mathrm{~mm}$. Following types of Terminals have been implemented:

- 3D-accelerometer Terminals with a size of $8 \times 10 \mathrm{~mm}$ are used to calculate the orientation of body segments by means of gravity vectors.

- General purpose ADC Terminal containing four analogdigital converters with a resolution of 10bit. Each ADC Terminal has a total size of $8 \times 8 \mathrm{~mm}$ and can be equipped with custom pull down resistors to be configured for specific measuring ranges. ADC Terminals are used to gather the temperature and resistance of the user's skin or light conditions.

- An I/O interface with four input buttons (e.g. for primitive posture class labeling) has four additional LEDs to signal events. The LEDs are currently configured to shine if a button is pressed.

\subsection{Gateways}

The main task of the Gateways is to provide an interface between the core system and remote Terminals. A special issue was the placement of the units on the garment. The goal was to permit a balanced distribution of Terminals over the whole body with a maximal cable length of $85 \mathrm{~cm}$. Two Gateways are located at the right and left upper arm to reach the upper body and limbs. A third Gateway was placed at the back in order to reach upper body locations and the head. A final Gateway was placed at the lower waist, to reach the legs. The position of the Gateways are indicated in Figure 3.

Each Gateway is equipped with four sockets, where Terminals can be connected. Hubs extend the number of Terminals attachable to a single Gateway to 127 . Hence, the system can be equipped with about 500 Terminals. We decided to mount a 3D-accelerometer on every Gateway, to give the core system a basic data gathering capability. The device is 
internally handled as a virtual acceleration Terminal. It is equipped with a MSP430F1611 16bit microcontroller, since it offers a good compromise between available computation power, necessary peripheral interfaces and power consumption. The selected model is equipped with $10 \mathrm{~KB}$ of RAM, which allows a later porting of an operating system.

Gateways and Konnex are connected by a 4 -wire Serial Peripheral Interface (SPI) bus in a redundant star topology. The Konnex, as the logical bus master, is able to detect broken signal wires and to restore the connection to unreachable devices via an associated Gateway in a static routing.

\subsection{Konnex}

The Konnex is the system master for communication, power and data processing. The latter is done by an additional MSP430 microprocessor. For reasons of comfort, the Konnex and the battery are located near to the body's center of mass, at the lower back where the extra weight is hardly noticeable for the wearer.

A central power supply generates a system-wide distributed voltage of $3.3 \mathrm{~V}$. It is sourced by a flat, detachable lithium polymer battery. A virtual Terminal is located on the Konnex in order to observe the system voltage. SMASH disables all communication modules and Terminals if the system voltage drops below a critical level. We performed a long term test to estimate the system's runtime. SMASH was equipped with three acceleration Terminals and performed an online classification of three randomly trained posture classes. The results were sent continuously to an outer host $\mathrm{PC}$ via the integrated Bluetooth module. For three runs, we measured a battery life far in excess of 14 hours.

\subsection{Textile Integration}

A challenge was to integrate the plain core system into the inside of a long sleeve garment, see Figure 1. Our goal was to keep the electronic- equipped garment comfortable, as it should not hinder any movement of the wearer. Also, the electronic components shall need to be kept save from environmental stress, technical shock, vibration and electrical shorts. While there exists a variety of integration methods, we decided to glue the core system into the inner side of the garment with silicone gel (polymerized siloxanes, see also Figure 4). The usage of silicone as a base material has several advantages:

- Low toxicity

- Electrically insulated

- Thermally stable

- Low chemical reactivity, resistant to oxygen and ozone

\section{SYSTEM CHARACTERISATION}

The ability of SMASH to distinguish different postures depends on several factors, listed in Table 1. The following section investigates the limits of the system's accuracy and presents the results of a study.

\subsection{Characterization of Accelerometer}

The accuracy of the system is limited by the electrical properties of the used ADXL330 accelerometer. The output of

\begin{tabular}{|c|c|c|}
\hline Time scale & Cause & Error \\
\hline Seconds & $\begin{array}{l}\text { Short term } \\
\text { signal drift }\end{array}$ & $\begin{array}{c}\text { Disturbance of measure- } \\
\text { ments in sensor space }\end{array}$ \\
\hline Seconds & Movements & $\begin{array}{l}\text { Shift of garment } \\
\text { relative to the body }\end{array}$ \\
\hline Hours & $\begin{array}{l}\text { Pull on/off } \\
\text { textile }\end{array}$ & Misalignment of sensors \\
\hline Years & $\begin{array}{l}\text { Deterioration } \\
\text { of sensors }\end{array}$ & $\begin{array}{l}\text { Malfunction or } \\
\text { failure of sensors }\end{array}$ \\
\hline
\end{tabular}

Table 1: Summary of systematic errors.

each Terminal's $\mathrm{X}, \mathrm{Y}$ and $\mathrm{Z}$ axis was measured three times for $-1 \mathrm{~g}, 0 \mathrm{~g}$ and $1 \mathrm{~g}$, respectively. All acceleration Terminals showed a similar accuracy for the different axes. Table 2 shows the average derivations for the outputs of the axes (for clearness, the deviation was converted from $\mathrm{g}\left[\mathrm{m} / \mathrm{s}^{2}\right]$ to degrees $\left.\left[{ }^{\circ}\right]\right)$. The system's resolution is electrically limited to approx. $1^{\circ}$.

\begin{tabular}{|l|c|c|c|}
\hline Axis & Min. Noise & Mean Noise & Max. Noise \\
\hline \hline $\mathrm{X}$ & $1.2^{\circ}$ & $1.3^{\circ}$ & $1.3^{\circ}$ \\
\hline $\mathrm{Y}$ & $0.8^{\circ}$ & $1.1^{\circ}$ & $1.7^{\circ}$ \\
\hline $\mathrm{Z}$ & $0.4^{\circ}$ & $0.8^{\circ}$ & $1^{\circ}$ \\
\hline
\end{tabular}

Table 2: Stability of the Terminals output, averaged for three readings for $-90^{\circ}, 0^{\circ}$ and $90^{\circ}$.

\subsection{Vertical Angle Resolution of the System}

The accuracy of the overall system was evaluated in a study. We picked a typical movement, like the abduction of the right arm, and cut it into equidistant steps of 5 degrees. The movement can be exercised in a range from approx. $0^{\circ}$ to $180^{\circ}$, which ends up in 37 different posture classes. For orientation, we printed a poster showing a semicircle and excentric beams in an respective angle of 5 degrees. Two female and three male subjects put on SMASH and were equipped with an acceleration Terminal at the right wrist. Subjects were instructed to stand with their back to the poster so that the shoulder joint was aligned in front of the semicircle's center (see Figure 5). Starting with pointing to the bottom, the right arm was abducted in steps of 5 degrees from $0^{\circ}$ to $180^{\circ}$. Each of the 37 postures was held for at least one second and labeled by an assistant. The whole exercise was repeated three times. The subjects were asked to perform random activities between the repetitions in order to realign the garment in a natural way.

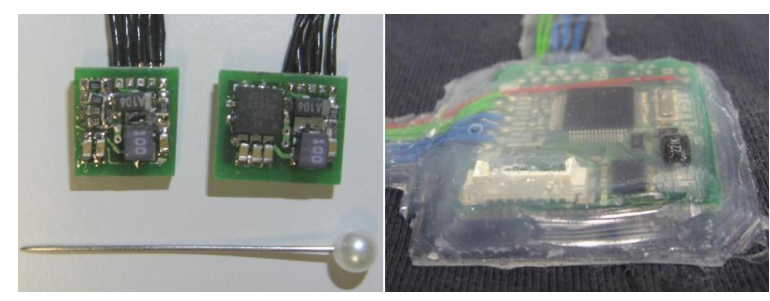

Figure 4: The left picture shows an ADC Terminal $(8 \times 8 \mathrm{~mm})$ and an acceleration Terminal $(8 \times 10 \mathrm{~mm})$. On the right, a silicone integrated Gateway is shown. 


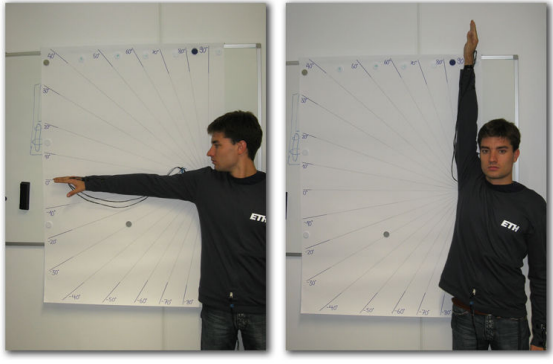

Figure 5: Experimental procedure for the system evaluation.

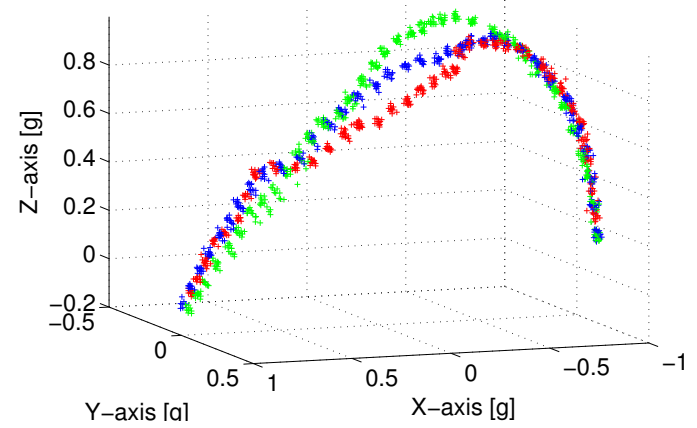

Figure 6: Three different repetitions of the exercise, performed by an exemplary user.

After all feature sets were recorded, we tried to discriminate the posture classes with Naive Bayes classification. The classifier was trained and tested with the raw sensor data of the right Gateway and the acceleration Terminal at the right wrist. A user-specific accuracy was computed with a 3-fold cross validation, performed for every repetition of the exercise. Considering all 37 classes, an average accuracy of $41 \%$ was achieved. By looking at the classified postures we noticed that only neighboring classes (abduction angles) are confused. When the classification was repeated for angles of $10^{\circ}$ (19 classes), an average user-specific accuracy of $85 \%$ was achieved. One reason for the low accuracy is the shift of the garment during the exercise. The user-specific accuracy is increased from $41 \%$ to $61 \%$ if the textile is locally fixed on the forearm. A more important reason is an observed rotation of the forearm during the exercises. Figure 6 shows the unit vectors for each 3 repetitions, for a randomly chosen subject. The rotation is indicated by the excursion of the Y-acceleration.

For a quantization of this effect, we calculated the absolute difference of the gravity vectors for the exercise's repetitions. Figure 7 shows the average orientation difference (error), depending on the arm's abduction angle (dotted line). The rotation reaches a maximum of about $20^{\circ}$ for a flexion of approx. $105^{\circ}$ and decreases to $3^{\circ}$ if the arm points up. This effect can be explained in two ways. On one hand the sleeve is shifted, if the arm is raised. It becomes tight fitting and aligns the acceleration Terminal in a similar way to the body. On the other hand the influence of the arm's rotation decreases if the arm is raised. In case the arm points up, the

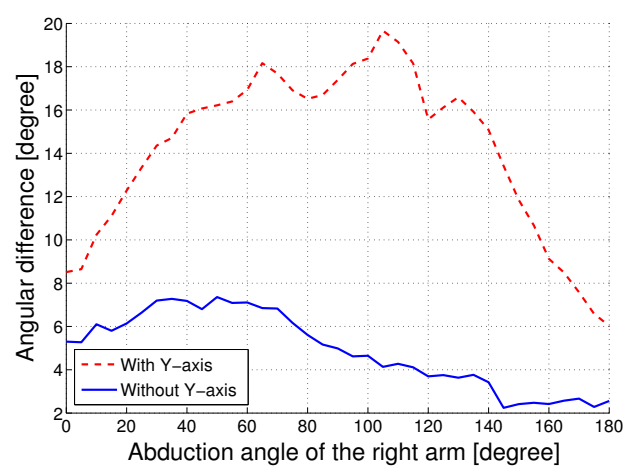

Figure 7: Average error in the orientation of the arm during the repetition of the experiment.

gravity vector is orthogonal to the rotation-sensitive $\mathrm{Y}$-axis.

The solid line in Figure 7 depicts the averaged angular deviations, if the Y-axis is masked. Hence, the system's average angular accuracy in the poster's plane is better than $7.5^{\circ}$. The classification was repeated without both Y-axes in order to avoid the rotation's influence. A final user-specific accuracy of $88 \%$ was reached.

\section{POSTURE CLASSIFICATION FOR RE- HABILITATION}

We conducted a study to evaluate the SMASH garment platform's feasibility for movement rehabilitation. Specifically, we concentrated on postures of the arms, relevant for the therapy of shoulder and elbow joints. The investigation of the system's stable operation and the textile fitting impact was conducted with healthy users. Here, we present the experimental procedure and the classification analysis results.

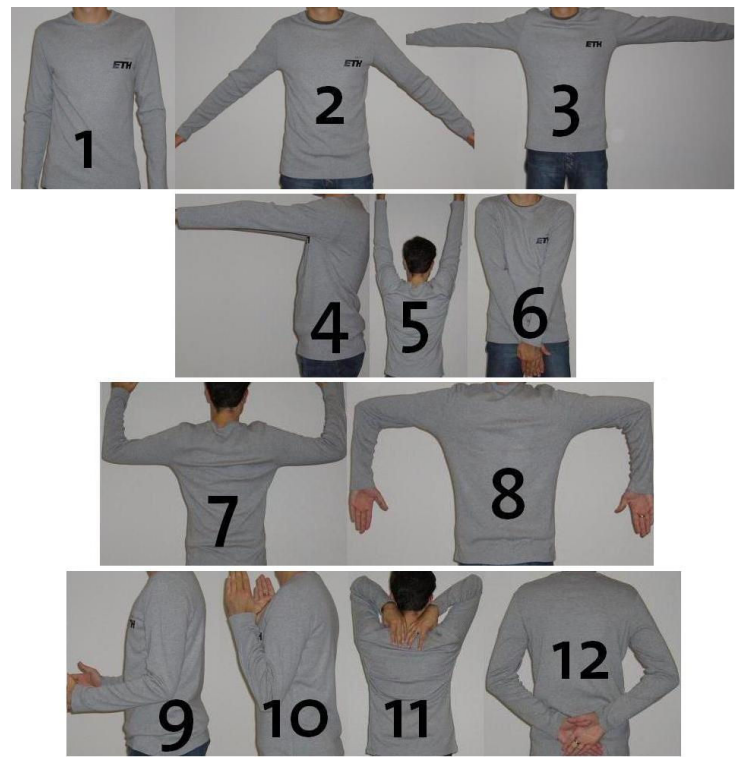

Figure 8: All twelve classified postures. 


\subsection{Experimental Procedure}

Eight users ( 3 female, 5 male) aged between 23 and 32 years participated in the study. The users wore the SMASH system (size: large) with one sensing acceleration Terminal placed at the end of the right sleeve. The Terminal was attached to the Gateway at the right upper arm. Both Terminal and Gateway sampled their 3D-accelerometer with $16 \mathrm{~Hz}$. For the purpose of this investigation the raw sensor data was transmitted from the Konnex to a remote PC for offline analysis. Figure 9 shows the sensor positioning. A set of exercises, commonly used for rehabilitation training of the shoulder and elbow joint was selected for the investigation. Table 3 summarizes the individual postures. Each exercise begins with the user standing upright, arms relaxed. This is indicated as normal position (class 1 in Tab. 3). Figure 8 shows all 12 postures, each was explained and shown to the users before the respective exercise. A large mirror was provided to the users, in order to verify the execution. Each user conducted three repetitions of all exercises. Between each repetition, users were asked to perform some random movements to restore the garment's natural alignment. An experiment observer annotated the individual postures for the following analysis. In total, 24 instances from 12 different postures were recorded.

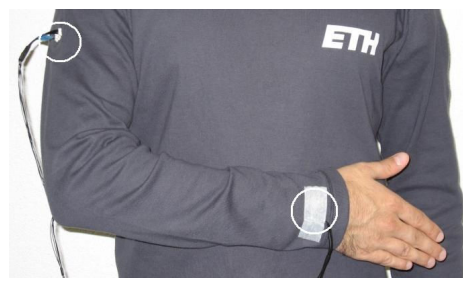

Figure 9: Sensor placement at the wrist and upper arm (marked with a circle).

\begin{tabular}{|c|c|c|}
\hline Class & Posture & Joint \\
\hline 1 & Normal & Combined \\
\hline \multicolumn{3}{|c|}{ Exercise 1: Abduction of arm } \\
\hline 2 & Abduction $40^{\circ}$ & Shoulder \\
\hline 3 & Abduction $90^{\circ}$ & \\
\hline \multicolumn{3}{|c|}{ Exercise 2: Flexion, Elevation of arm } \\
\hline 4 & Flexion $90^{\circ}$ & Shoulder \\
\hline 5 & Elevation $170^{\circ}$ & \\
\hline \multicolumn{3}{|c|}{ Exercise 3: $15^{\circ}$ Adduction of arm } \\
\hline 6 & Adduction $15^{\circ}$ & Shoulder \\
\hline \multicolumn{3}{|c|}{ Exercise 4: Rotation of arm } \\
\hline 3 & Abduction $90^{\circ}$ & Shoulder \\
\hline 7 & Rotation $90^{\circ}$ in & \\
\hline 8 & Rotation $90^{\circ}$ out & \\
\hline \multicolumn{3}{|c|}{ Exercise 5: Flexion of forearm } \\
\hline 9 & Flexion $90^{\circ}$ & Elbow \\
\hline 10 & Flexion $130^{\circ}$ & \\
\hline \multicolumn{3}{|c|}{ Exercise 6: Neck-grip } \\
\hline 11 & Neck-grip & Combined \\
\hline \multicolumn{3}{|c|}{ Exercise 7: Flexion of forearm } \\
\hline 12 & Skirt-grip & Combined \\
\hline
\end{tabular}

Table 3: Rehabilitation exercises included in the study. Each exercise consists of one or several postures.

\subsection{Classification Procedure}

Using the recorded datasets a classification analysis was performed. We utilized a Nearest Centroid algorithm and a cross-validation scheme to split training and testing instances for our evaluation. The mean accelerometer readings from upper arm and wrist were used as classification features.

We analyzed the classification performance for the three training modes: user-specific, user-adapted and user-independent. For the user-specific evaluation, training and testing was performed on the posture instances from each user individually. A leave-one-out cross-validation was used. In user-adapted mode posture instances from all users were selected for training and testing set. A three-fold crossvalidation was used. The user-adapted evaluation includes instances from more than one subject and is an intermediate step between user-specific and user-independent modes. The user-independent is typically the hardest test. Here, the classification performance is analyzed for postures from a user who's postures were not included in the algorithm training. This test indicates the performance of the system when used with new person. For this mode, a cross-validation on the number of users (eight) was used.

\subsection{Classification Results}

The classification results for all three training modes were: $95 \%$ for user-specific, $94 \%$ for user-adapted and $89 \%$ for user-independent. The best rate was clearly achieved for the user-specific mode. However, the classification performs only slightly better compared to the user-adapted case.

For the user-independent mode training was performed on the data from other users. Here the performance is lower, indicating differences in the postures between the users. Figure 10 depicts the performances of all training modes along with minimum and maximum values. The values indicate the range of results for each user in the user-specific and user-independent evaluations. For the user-adapted case, the result variance from the cross-validation folds is shown. As expected, this variance is very low, since instances from all users are used for training and testing in this mode.

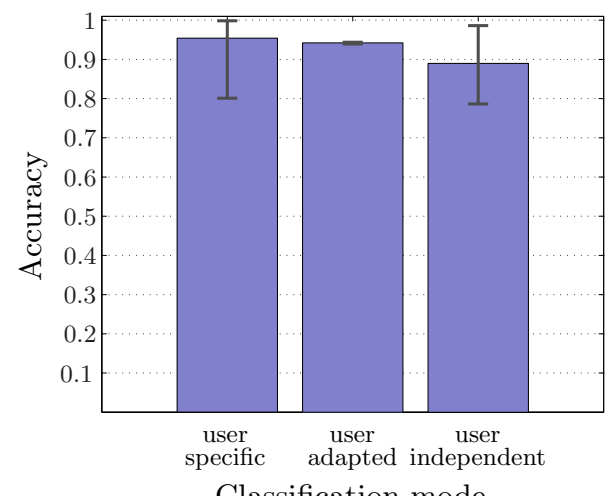

Classification mode

Figure 10: Posture classification accuracy for the rehabilitation exercises and the three training modes: user-specific, user-adapted and user-independent. 
Figures 11 and 12 show the classifier confusion matrices for the user-specific and user-independent cases respectively. This class confusion provides an indication on the misclassified postures. The overall good classification of each class is shown by the main diagonal being close to one. Confusions (all fields besides the main diagonal) for both training modes are found for posture classes 3 and 4 as well as classes 1 and 6 . This effect can be explained by the visual similarity of the postures (compare Figure 8). For the userindependent evaluation further confusions were observed.

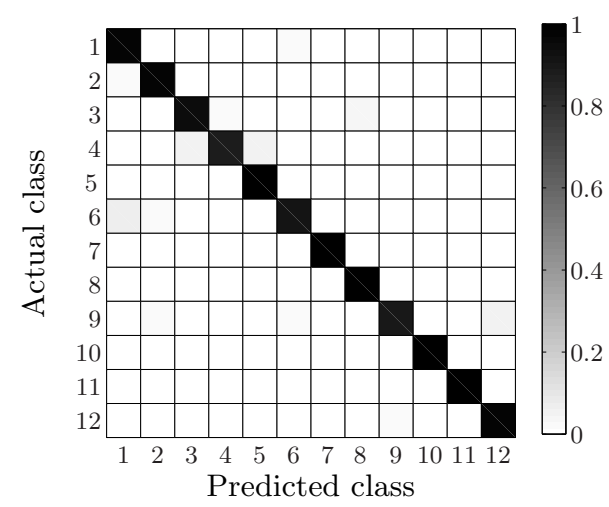

Figure 11: Posture classification confusion matrix for the rehabilitation exercises in user-specific training mode.

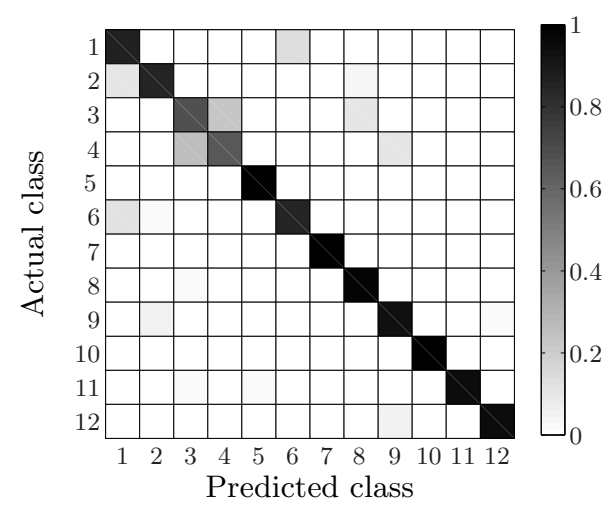

Figure 12: Posture classification confusion matrix for the rehabilitation exercises in user-independent training mode.

In conclusion, the rehabilitation exercises can be successfully classified with good accuracy, even for the more challenging user-adapted and user-independent training modes. This indicates that the movement of the textile does not prevent the accurate classification of the selected exercises with simple acceleration sensors.

\subsection{Influence of the Textile Fitting}

While good overall classification results for the 12 postures were achieved, we observed user dependent variations in the classification performance. We assumed that the performance could be partially explained by the user's body size and larger variations in the garment positioning during the exercised. In the experiments one garment model was worn by all users. We subsequently analyzed the userspecific classification performance in relation to the body dimensions (size and arm length).

We investigated the relationship between classification rate and the user arm length and body size. Contrary out initial assumption, we found no clear dependency between the variables. For only one user (with a height below $165 \mathrm{~cm}$ ) a noticeably reduced accuracy was observed.

The individual cross-validation runs for each user resulted in a varying classification performance. We assume that this variation is linked to the body dimensions. Indeed, Figure 13 supports this assumption: lower accuracy variations were found for both longer arm length and body size. While our evaluation set of eight users is not sufficient to infer a conclusive rule, this relation is very intuitive.
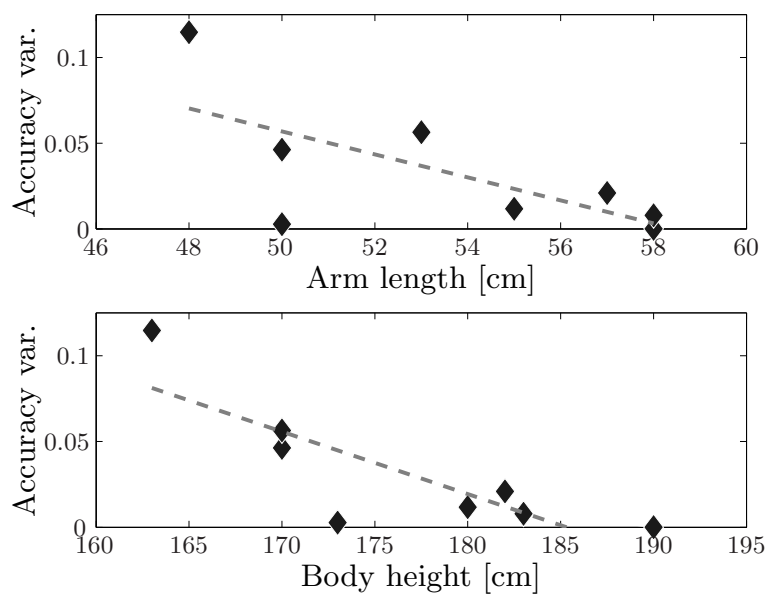

Figure 13: User-specific classification accuracy variation (maximum-minimum) in relation to user arm length and body size. The dashed line indicates the result of a linear fitting.

\section{CONCLUSIONS}

In this work we presented SMASH, a novel sensing and processing platform integrated into a upper body garment. The system processes sensor data in a three-layer distributed architecture. At the signal level remote sensing terminals are used, features are extracted by local gateways and a central system master performs classification tasks. Sensor data processing and classification as well as hot-plug-capabilities were implemented in the system. In our current evaluation, accelerometer sensors were used. However, the system is designed to support different sensing modalities.

We evaluated the system performance in two independent studies. The first evaluation addressed the sensor resolution in a characterization experiment with five users. We found that the system was able to detect angle changes at a resolution of $7.5^{\circ}$. This result was confirmed by posture classification in $5^{\circ}$ and $10^{\circ}$ resolution. The procedure has potential for the validation of similar textile systems in the future. 
In the second evaluation, we studied the classification of rehabilitation exercises. Eight users conducted 12 individual postures from different exercises while wearing the garment. The feasibility of this application was investigated by a cross-validation classification scheme in different training modes. We obtained classification rates of $95 \%$ for the user-specific and $89 \%$ for the user-independent mode. These good results indicate that the textile system can be used successfully to monitor the investigated rehabilitation exercises. Potential applications include tele-rehabilitation, where the therapist is not locally available to observe or verify the exercise execution. Concepts for these applications are currently developed, e.g. by the Special Interest Group on Telerehabilitation [15].

Moreover, we analyzed the relation between user's dimensions and the classification performance. Visual analysis of scatter plots indicated that the variations in classification performance were linked to the user's body size and arm length. For smaller users, larger performance variations were found. However, it is clear that many further garment fitting aspects could influence the classification performance of exercise postures.

While our current results are already very promising for rehabilitation and sports applications, we plan to extend the studies to more subjects and postures. We will further investigate the relation of fitting aspects on the feasibility of the garment. Moreover, we plan to extend the platform with additional sensing modalities through our plug-and-play design. To this end, we will release the system as a community platform to advance further research in the field of textile integrated electronics.

\section{ACKNOWLEDGMENTS}

The authors are grateful to Urs Egger and Elias Bürli for their support in building the textile prototype and Corina Schuster, Reha Rheinfelden, for specifying the rehabilitation exercises used in this work. The project was partly supported by the Swiss State Secretariat for Education and Research.

\section{REFERENCES}

[1] J. K. Perng, B. Fisher, Seth Hollar and K. S. J. Pister. Acceleration Sensing Glove (ASG). In Proceedings of the 3rd IEEE International Symposium on Wearable Computers (ISWC), pages 178-180, 1999

[2] A. Tognetti, F. Lorussi, R. Bartalesi, S. Quaglini, M. Tesconi, G. Zupone and D. De Rossi. Wearable kinesthetic system for capturing and classifying upper limb gesture in post-stroke rehabilitation. In Journal of NeuroEngineering and Rehabilitation 2005, 2:8 doi:10.1186/1743-0003-2-8

[3] A. Just, Y. Rodriguez and S. Marcel. Hand Posture Classification and Recognition using the Modified Census Transform. In 7th International Conference on Automatic Face and Gesture Recognition, 2006, pages 351-356

[4] T. Starner and A. Pentland. Real-Time American Sign Language Recognition From Video Using Hidden Markov Models. SCV95, 5B Systems and Applications, 1995
[5] S. Park, K. Mackenzie, and S. Jayaraman. The wearable motherboard: A framework for personalized mobile information processing (PMIP). Presented at the ACM/IEEE 39th Design Automation Conf., New Orleans, 2002

[6] L. E. Dunne, P. Walsh, B. Smyth and B.Caulfield. Design and Evaluation of a Wearable Optical Sensor for Monitoring Seated Spinal Posture. In Proceedings of the 10th IEEE International Symposium on Wearable Computers, Oct. 2006, pages 65-68

[7] T. Giorgino, F. Lorussi, D. De Rossi and S. Quaglini. Posture Classification via Wearable Strain Sensors for Neurological Rehabilitation. Engineering in Medicine and Biology Society 2006, Aug. 2006 ,pages: 6273-6276

[8] C. Mattmann, O. Amft, H. Harms and G. Tröster. Recognizing Upper Body Postures using Textile Strain Sensors. In Proceedings of The 11th International Symposium on Wearable Computers, Boston, October 2007, pages 29-36

[9] H. Junker, P. Lukowicz and Gerhard Tröster. PadNET: Wearable Physical Activity Detection Network. In Proceedings of the 7th IEEE International Symposium on Wearable Computers, 2003, page 244

[10] Moven - wireless inertial motion capture. www.moven.com

[11] E. R. Bachmann, X. Yun and R. B. McGhee. Sourceless tracking of human posture using small inertial/magnetic sensors. In Proceedings of Computational Intelligence in Robotics and Automation, 2003, pages 822-829

[12] J. Marins, X. Yun, E. R. Bachmann, R. McGhee and M. Zyda. An Extended Kalman Filter for Quaternion-Based Orientation Estimation Using MARG Sensors., 2001

[13] D. Roetenberg, H. Luinge and P. Veltink. Inertial and magnetic sensing of human movement near ferromagnetic materials. In Proceedings of the 2nd IEEE and ACM International Symposium on Mixed and Augmented Reality, 2003, page 268

[14] B. Clarkson, K. Mase and Alex Pentland. Recognizing User Context via Wearable Sensors. In Proceedings of the 4th IEEE International Symposium on Wearable Computers (ISWC), 2000, pages 69-76

[15] Special Interest Group on Telerehabilitation http://www.telerehabilitation.net/index.html 